\title{
Unilateral Verrucous Psoriasis
}

\author{
Riana D. Sanyal, MD; Nikki S.Vyas, MD; Allen N. Sapadin, MD; Robert G. Phelps, MD
}

\section{PRACTICE POINTS}

- Verrucous psoriasis is a rare variant of psoriasis characterized by hypertrophic verrucous papules and plaques on an erythematous base.

- Histologically, verrucous psoriasis presents with overlapping features of verruca and psoriasis.

- Although psoriasis typically presents in a symmetric distribution, unilateral psoriasis can occur either de novo in younger patients or after surgical trauma in older patients.
Verrucous psoriasis is a variant of psoriasis that presents with wartlike clinical features and overlapping histologic features of verruca and psoriasis. The disease typically arises in patients with established psoriasis but can occur de novo. We report the case of an 80-year-old man with a history of hypertension and coronary artery disease who presented with a rash characterized by multiple asymptomatic plaques with overlying verrucous nodules on the left side of the body. The lesions appeared shortly after coronary artery bypass surgery with a saphenous vein graft.

Cutis. 2021;107:97-98.

\section{Case Report}

An 80-year-old man with a history of hypertension and coronary artery disease presented to the dermatology clinic with a rash characterized by multiple asymptomatic plaques with overlying verrucous nodules on the left side of the abdomen, back, and leg (Figure 1). He reported that these "growths" appeared 20 years prior to presentation, shortly after coronary artery bypass surgery with a saphenous vein graft. The patient initially was given a diagnosis of verruca vulgaris and then biopsy-proven psoriasis later that year. At that time, he refused systemic treatment and was treated instead with triamcinolone acetonide ointment, with periodic surgical removal of bothersome lesions.

At the current presentation, physical examination revealed many hyperkeratotic, yellow-gray, verrucous nodules overlying scaly, erythematous, sharply demarcated plaques, exclusively on the left side of the body, including the left side of the abdomen, back, and leg.
The differential diagnosis included linear psoriasis and inflammatory linear verrucous epidermal nevus (ILVEN).

Skin biopsy showed irregular psoriasiform epidermal hyperplasia with acanthosis, hyperkeratosis, and papillomatosis, with convergence of the rete ridges, known as buttressing (Figure 2A). There were tortuous dilated blood vessels in the dermal papillae, epidermal neutrophils at the tip of the suprapapillary plates, and Munro microabscesses in the stratum corneum (Figure 2B). Koilocytes were absent, and periodic acid-Schiff staining was negative. Taken together, clinical and histologic features led to a diagnosis of unilateral verrucous psoriasis.

\section{Comment}

Presentation and Histology-Verrucous psoriasis is a variant of psoriasis that presents with wartlike clinical features and overlapping histologic features of verruca and
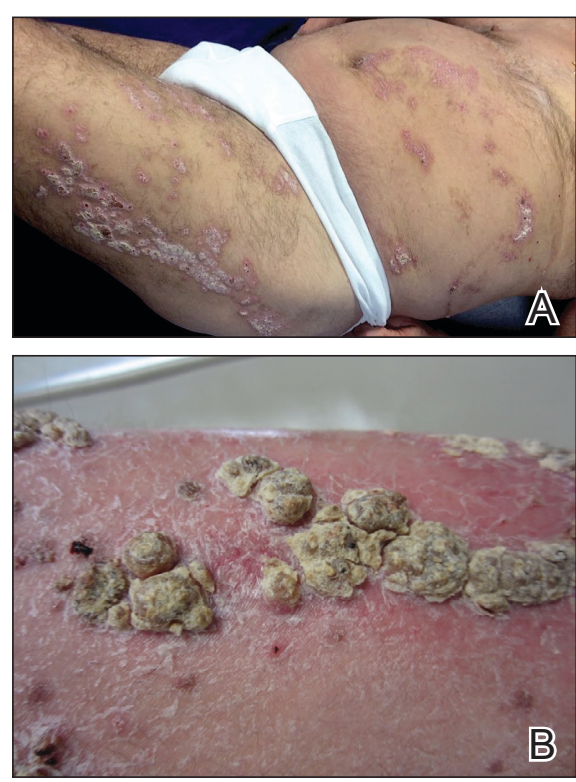

FIGURE 1. Verrucous psoriasis on the left side of the body. A, Welldemarcated, scaly, erythematous plaques. B, Hyperkeratotic verrucous growths.

From the Department of Dermatology, Icahn School of Medicine at Mount Sinai, New York, New York.

The authors report no conflict of interest.

Correspondence: Riana D. Sanyal, MD, Icahn School of Medicine at Mount Sinai, 1 Gustave L. Levy PI, New York, NY 10029

(riana.dutt@icahn.mssm.edu).

doi: $10.12788 /$ cutis. 0174 
FIGURE 2. Histopathology of verrucous psoriasis. A, Irregular psoriasiform epidermal hyperplasia with acanthosis, hyperkeratosis, papillomatosis, and buttressing (converging to the center) of rete ridges (H\&E, original magnification $\times 20$ ). $B$, Tortuous dilated vessels were present on a biopsy specimen in dermal papillae, along with epidermal neutrophils that surmount the tips of suprapapillary plates. Intracorneal Munro microabscesses also were present $(\mathrm{H} \& \mathrm{E}$, original magnification $\times 100$ ).
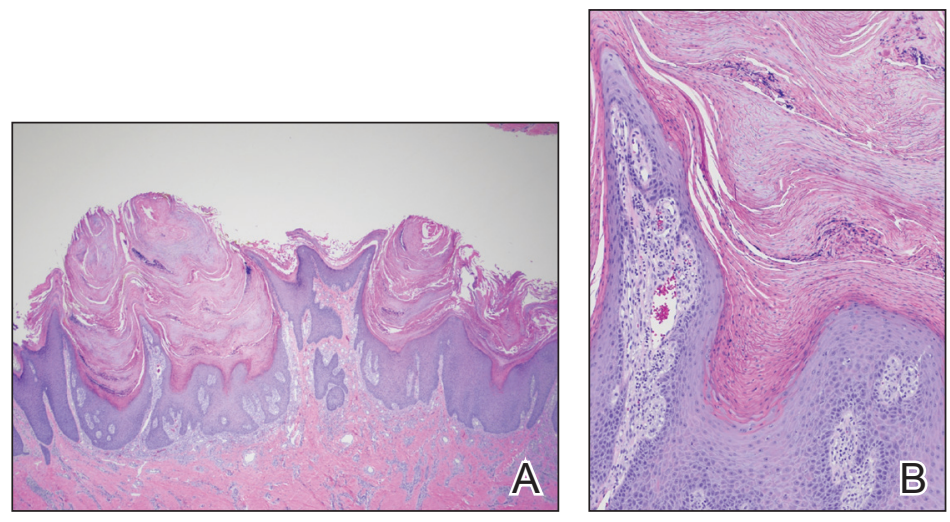

psoriasis. It typically arises in patients with established psoriasis but can occur de novo.

Histologic features of verrucous psoriasis include epidermal hyperplasia with acanthosis, papillomatosis, and epidermal buttressing. ${ }^{1}$ It has been hypothesized that notable hyperkeratosis observed in these lesions is induced by repeat trauma to the extremities in patients with established psoriasis or by anoxia from conditions that predispose to poor circulation, such as diabetes mellitus and pulmonary disease. ${ }^{1,2}$

Pathogenesis-Most reported cases of verrucous psoriasis arose atop pre-existing psoriasis lesions. ${ }^{3,4}$ The relevance of our patient's verrucous psoriasis to his prior coronary artery bypass surgery with saphenous vein graft is unknown; however, the distribution of lesions, timing of psoriasis onset in relation to the surgical procedure, and recent data proposing a role for neuropeptide responses to nerve injury in the development of psoriasis, taken together, provide an argument for a role for surgical trauma in the development of our patient's condition.

Treatment-Although verrucous psoriasis presents both diagnostic and therapeutic challenges, there are some reports of improvement with topical or intralesional corticosteroids in combination with keratolytics, ${ }^{3}$ coal tar, ${ }^{5}$ and oral methotrexate. ${ }^{6}$ In addition, there are rare reports of successful treatment with biologics. A case report showed successful resolution with adalimumab, ${ }^{4}$ and a case of erythrodermic verrucous psoriasis showed moderate improvement with ustekinumab after other failed treatments. ${ }^{7}$

Differential Diagnosis_-Psoriasis typically presents in a symmetric distribution, with rare reported cases of unilateral distribution. Two cases of unilateral psoriasis arising after a surgical procedure have been reported, one after mastectomy and the other after neurosurgery. 8,9 Other cases of unilateral psoriasis are reported to have arisen in adolescents and young adults idiopathically.

A case of linear psoriasis arising in the distribution of the sciatic nerve in a patient with radiculopathy implicated tumor necrosis factor $\alpha$, neuropeptides, and nerve growth factor released in response to compression as possible etiologic agents..$^{10}$ However, none of the reported cases of linear psoriasis, or reported cases of unilateral psoriasis, exhibited verrucous features clinically or histologically. In our patient, distribution of the lesions appeared less typically blaschkoid than in linear psoriasis, and the presence of exophytic wartlike growths throughout the lesions was not characteristic of linear psoriasis.

Late-adulthood onset in this patient in addition to the absence of typical histologic features of ILVEN, including alternating orthokeratosis and parakeratosis, ${ }_{1}^{11}$ make a diagnosis of ILVEN less likely; ILVEN can be distinguished from linear psoriasis based on later age of onset and responsiveness to antipsoriatic therapy of linear psoriasis. ${ }^{12}$

\section{Conclusion}

We describe a unique presentation of an already rare variant of psoriasis that can be difficult to diagnose clinically. The unilateral distribution of lesions in this patient can create further diagnostic confusion with other entities, such as ILVEN and linear psoriasis, though it can be distinguished from those diseases based on histologic features. Our aim is that this report improves recognition of this unusual presentation of verrucous psoriasis in clinical settings and decreases delays in diagnosis and treatment.

\section{REFERENCES}

1. Khalil FK, Keehn CA, Saeed S, et al.Verrucous psoriasis: a distinctive clinicopathologic variant of psoriasis. Am J Dermatopathol. 2005;27:204-207.

2. Wakamatsu K, Naniwa K, Hagiya Y, et al. Psoriasis verrucosa. J Dermatol. 2010;37:1060-1062.

3. Monroe HR, Hillman JD, Chiu MW. A case of verrucous psoriasis. Dermatol Online J. 2011;17:10.

4. Maejima H, Katayama C, Watarai A, et al. A case of psoriasis verrucosa successfully treated with adalimumab. J Drugs Dermatol. 2012;11:E74-E75.

5. Erkek E, Bozdoğan O. Annular verrucous psoriasis with exaggerated papillomatosis. Am J Dermatopathol. 2001;23:133-135.

6. Hall L, Marks V, Tyler W. Verrucous psoriasis: a clinical and histopathologic mimicker of verruca vulgaris. J Am Acad Dermatol. 2013;68(4 suppl 1):AB218.

7. Curtis AR, Yosipovitch G. Erythrodermic verrucous psoriasis. J Dermatolog Treat. 2012;23:215-218.

8. Kim M, Jung JY, Na SY, et al. Unilateral psoriasis in a woman with ipsilateral post-mastectomy lymphedema. Ann Dermatol. 2011;23(suppl 3):S303-S305.

9. Reyter I, Woodley D. Widespread unilateral plaques in a 68-year-old woman after neurosurgery. Arch Dermatol. 2004;140:1531-1536.

10. Galluzzo M, Talamonti M, Di Stefani A, et al. Linear psoriasis following the typical distribution of the sciatic nerve. J Dermatol Case Rep. 2015;9:6-11.

11. Sengupta S, Das JK, Gangopadhyay A. Naevoid psoriasis and ILVEN: same coin, two faces? Indian J Dermatol. 2012;57:489-491.

12. Morag C, Metzker A. Inflammatory linear verrucous epidermal nevus: report of seven new cases and review of the literature. Pediatr Dermatol. 1985;3:15-18. 